\title{
DEL UTILITARISMO A LA ÉTICA
}

$Y$ LOS PRINCIPIOS: INDISPENSABLES

EN LOS MODELOS ECONÓMICOS

\author{
Julio Cesar Montoya Rendón ${ }^{1}$ \\ José Luis Montaño Hurtado²
}

Recibido: 15 de mayo de 2013 Aceptado: 12 de agosto de 2013

"La ética es, en cierta medida, como el

oxígeno: sólo nos damos cuenta de su

importancia cuando nos falta"

A. Sen

\section{Resumen}

El utilitarismo enuncia: «mayor bien para el mayor número de personas», eset concepto tratado por Mill, Smith yKeynes, entre otros, está ausente de los actuales modelos económicos orientados más a la acumulación de riqueza a ultranza que a la felicidad del hombre y el bienestar social.

La problemática contemporánea plantea una crisis de ética y principios, lo que lleva a preguntar: ¿Más que crísis económicas, sociales y políticas: hay crísis de valores y de principios éticos? pregunta desencadenante de otras y de objeciones a los modelos económicos imperantes. Pregunta que traza el objetivo de esta reflexión: ilustrar cono los intereses particulares, codiciosos y faltos de ética, desdibujan el sentido de bienestar que en general enuncian los modelos económicos.

Asociado el concepto utilitarista al de Responsabilidad Social Empresarial - RSE - como una opción dinamizadora, en la búsqueda del bien y el beneficio para todos los grupos de interés organizacional, se encuentra que esta RSE, en la forma que es gestionada, no tiene un sentido altruista por parte de las organizaciones, sino simplemente como otra unidad de negocio.

Es inevitable concluir: la ética y los principios es indispensables en la aplicación de los modelos económicos para que éstos cumplan sus propósitos.

Palabras clave: Utilitarismo, ética, principios, modelos económicos

\footnotetext{
${ }^{1}$ Administrador de Empresas, Especialista en Pedagogía para el Desarrollo del Aprendizaje Autónomo y MBA. Líder Ecacen Cead Palmira.

${ }^{2}$ Administrador de Empresas, Especialista en Pedagogía para el Desarrollo del Aprendizaje Autónomo, MBA, candidato a doctor en Administración. Director Zona Centro Sur de la Unad UNAD.
} 


\section{THE ETHICS OF UTILITARIANISM AND PRINCIPLES: ESSENTIAL IN ECONOMIC MODELS}

"Ethics is, to some extent, like oxygen: we only realize its importance when we lack"

A. Sen

\section{Abstract}

Utilitarianism states: "greatest good for the greatest number of people" concept discussed by Mill, Smith, Keynes, among others. It is clear that it is absent of the current economic models faced more to the accumulation of wealth at any cost than to the man's happiness and welfare.

The contemporary problematic raises crisis of ethics and principles, what he leads to asking: More than economic, social and political crises: is there crisis of values and of ethical principles? Unchaining question of others and of objections to the prevailing economic models. Question, that plans the aim of this reflection: Illustrate cone particular interests, greedy and unethical, blur the sense of well being forth the economic models.

Associated utilitarian concept Corporate Social Responsibility - CSR - as a motivator option in the search for the good and benefit to all stakeholders in an organization, you will find that this CSR, in the way it is managed, not altruistic sense of the organizations, but simply as another business unit.

It's inevitable conclusion: the ethics and principles are essential in the application of economic models for them to be viable

Keywords: utilitarianism, ethics, principles, economic models

\section{Introducción}

Desplome de la URSS, se cuestiona el Euro, caen hipotecas en EEUU, crisis política en Venezuela, Colombia país con mayor desigualdad en el mundo, hambruna en África, primavera Árabe, carrusel de la contratación, crecimiento económico a ultranza y sin sentido, burbuja financiera estalla, crisis inmobiliaria en España, utilidades ofensivas del sector financiero en Colombia, Estado colombiano apalanca sector financiero, privatizaciones cunden, aumenta el desempleo, inestabilidad laboral, consumismo aumenta, aumenta la concentración de la riqueza, desabastecimiento en Venezuela, globalización se impone, matan sindicalista, se dispara la corrupción, compra de votos, solidaridad a merced del individualismo, Corea del Norte amenaza a Corea del Sur, se impone dictadura del mercado, privatizan empresas públicas, atacan periodistas, presos políticos en Cuba, expropiaciones en Argentina, crisis ambiental en el mundo, China es la maquila del mundo.

Las frases anteriores pueden ser un pequeño collage de los titulares de los diarios de los últimos 35 años en todos los países, bueno, al menos en aquellos donde no ha habido o no hay censura de prensa. 
Lo anterior hace pensar que el neoliberalismo a ultranza y el socialismo totalitario (incluyendo todos sus matices) son dos caras de la misma moneda: crisis económica, social, política y desigualdad.

Para tocar una pequeña arista en este contexto se podría preguntar, sólo para tocar un ejemplo, sobre las privatizaciones: ¿Si son buenos negocios para el capital privado, por qué no los son para el Estado? La contraparte podría preguntar: ¿Por qué en una economía planificada la productividad es mediocre?

Entonces: ¿El problema es de los modelos económicos? ¿Es de la gestión de los modelos económicos? ¿Es de quienes gestionan los modelos económicos? ¿Es apenas natural que se den este orden de cosas? Pues este "orden" de cosas lo sintetiza muy bien Stiglitz (2012) en una corta oración: «El I1\% de la población tiene lo que el $99 \%$ necesita». Es decir, este «orden» donde no aplica ni siquiera Pareto, se podría enunciar como «el mayor bien para el menor número de personas».

El planteamiento anterior, esboza el objetivo de esta reflexión: llustrar cono los intereses particulares, codiciosos y faltos de ética, desdibujan el sentido de bienestar que en general enuncian y pretenden los modelos económicos. Objetivo, que desde luego, anticipa respuestas a las preguntas formuladas anteriormente e indica el sentido crítico frente al ya mencionado «orden" que actualmente impera y gobierna todos los escenarios de actuación del hombre contemporáneo.

Aránzazu (1999), apoyado en los planteamientos de Amartya Sen, afirma que la economía siempre tuvo una conexión con la filosofía especialmente con la ética, lo que permitía una visión más compleja de la actividad humana vista de una manera holística e incluso altruista. Pero, paulatinamente la economía se fue apartando del sendero ético, en la medida que se hacía más técnica, al buscar propósitos de eficiencia, desarrollo y crecimiento material; el vacio que dejaba la ética se hizo más notorio.

Es claro que el distanciamiento de la economía de la ética, significa un distanciamiento del original principio utilitarista e individual que planteó Bentham y que retomó Mill como bien social, quien llega a afirmar que los intereses individuales se deben subordinar al bien general, según se explica más adelante. Pero el criterio técnico - económico se ha impuesto al criterio ético económico, lo que ha significado que se dé prioridad a la rentabilidad del capital, aunque este lo posea la minoría, sobre las oportunidades de trabajo, aunque esta potencialidad la tengan la mayoría de seres humanos.

\section{Contenido}

El utilitarismo parte de la premisa que dice: «El mayor bien para el mayor número de personas" (Priestly, citado por Anderson, 2000), frase reveladora para Bentham (1789) quien escribió:

«La naturaleza ha colocado a la humanidad bajo el gobierno de dos amos soberanos, el dolor y el placer. Les corresponde sólo a ellos señalar lo que debemos hacer, así como determinar lo que haremos. Por un lado, la norma del bien y del mal, por el otro la cadena de causas y efectos, están sujetos al trono de ellos. Nos gobiernan en todo lo que hacemos, en todo lo que decimos, en todo lo que pensamos; todo esfuerzo que hagamos para librarnos de nuestra sujeción servirá solo para demostrarla y confirmarla».

A su vez, el pensamiento de Bentham, se convertiría en la doctrina orientadora de John Stuart Mill, quien escribiría una obra titulada El Utilitarismo (1861) en la que señala que la búsqueda de la felicidad no es un propósito individual, sino social: 
«El credo que acepta la Utilidad o Principio de la Mayor Felicidad como fundamento de la moral, sostiene que las acciones son justas en la proporción con que tienden a promover la felicidad; e injustas en cuanto tienden a producir lo contrario de la felicidad. Se entiende por felicidad el placer, y la ausencia de dolor; por infelicidad, el dolor y la ausencia de placer» (Mill, 1861).

Mill, utiliza una concepción amplia del placer en el sentido que le da Epicuro: no es solamente el placer de los sentidos, es el placer del intelecto, del pensamiento y del obrar bien, es decir, en un sentido cristiano, atribuyéndole tanto valor a la calidad del placer como a la cantidad que era el principal atributo que le daba Bentham en su concepción cuantitativa de la felicidad.

Es importante resaltar la anterior concepción en términos de Mill:

«En la norma áurea de Jesús de Nazaret, leemos todo el espíritu de la ética utilitarista: Haz como querrías que hicieran contigo y ama a tu prójimo como a ti mismo. En esto consiste el ideal de perfección de la moral utilitarista. Lo que se puede entender como fuente de felicidad, hacer feliz a los demás, donde prevalecen los intereses generales sobre los particulares, o de otra manera, ajustar los propios intereses a los de los de la colectividad» (Mill, 1861).

La idea de Mill en que prevalece el interés general sobre el del individuo, encuentra un contraste con Adam Smith (1776), quien a propósito del individuo afirma «... pues al perseguir su "propio interés", promueve el de la sociedad de una manera más efectiva que si esto entrara en sus designio", de esta manera justifica la no intervención del Estado en los asuntos económicos, bajo la premisa de que hay una «mano invisible» que regula y equilibra las fuerzas del mercado, en otras palabras: laissez faire laissez passer. Convirtiéndose estos planteamientos en la orientación del liberalismo económico que, a su vez, adopta tres principios del utilitarismo que es importante recordar:

« La utilidad como principio político operativo que determina los fines colectivos, de acuerdo con la propiedad de cualquier objeto de producir beneficio, placer o felicidad, o evitar el mal o la infelicidad de aquella parte cuyo interés se está considerando.

- La felicidad como principio del individualismo, que obliga al gobernante a tener en cuenta los sentimientos de los individuos, y que se resuelve en un doble criterio de proporcionar una mayor felicidad a un mayor número de personas, y

- El placer como fin espontáneo de todo acto humano, siendo así que la felicidad se resuelve en una suma de placeres, y la moral se reduce al cálculo matemático que establece la bondad de una acción según el balance de placeres y penas» (Fontrodona, sin fecha).

Entonces, Smith encuentra razonable que cada individuo vaya en pos de su propio interés y al lograrlo, está aportando al interés de toda la sociedad y por consiguiente a su felicidad.

Por su parte, Keynes le da importancia a la intervención del Estado, al mercado en una justa medida o en la medida de lo necesario, bajo la siguiente observación:

«El problema político de la humanidad consiste en combinar tres cosas: eficiencia económica, justicia social y libertad individual. La primera necesita sentido, prudencia y conocimiento técnico; la segunda, un espíritu desinteresado y entusiasta, que ame al hombre corriente; la tercera, tolerancia, amplitud de miras, apreciación de las excelencias de la variedad y de la independencia, 
que prefiere, ante todo, dar oportunidades libres a lo excepcional y a lo ambicioso. El segundo ingrediente es el mejor patrimonio del gran partido del proletariado. Pero la primera y la tercera requieren las cualidades del partido que, por sus tradiciones y sus antiguas simpatías, ha sido el hogar del individualismo económico y de la libertad social» (EP, p. 313, citado por Crespo, 2004).

Ciertamente, el pensamiento de Keynes era altruista y en cierta medida, opuesto al utilitarismo del tipo cuantitativo planteado por Bentham y no del todo contrario al liberalismo económico, puesto que le daba importancia a la eficiencia económica y a la libertad individual. Una de las diferencias, es que plantea la necesidad de que el Estado promueva el pleno empleo, lo que, en si es «un argumento que trae también claras resonancias utilitaristas, propugna la satisfacción de los deseos, el incremento de los salarios, el aumento de los déficits presupuestarios como fuerzas generadoras del crecimiento" (Fontrodona, s.f.).

Es así como el utilitarismo ha cobrado una gran influencia en las teorías y modelos económicos que se han implantado alternativamente entre liberales e intervencionistas; especialmente en la segunda mitad del siglo XX y comienzos del $X X I$ en occidente y con mayor pragmatismo en las tres últimas décadas en los emergentes países asiáticos.

Teniendo en cuenta que estos modelos económicos utilitaristas han predominado en las últimas décadas y que en teoría promueven el bien y la felicidad para los seres humanos, ya sea para el individuo o para la sociedad en su conjunto, y que definitivamente existe una relación entre economía y felicidad (Giarrizzo, sin fecha), cabe preguntarse: ¿Por qué cada vez hay más millones de seres humanos por debajo de la línea de pobreza? ¿Por qué las diferencias entre ricos y pobres son cada vez mayores? ¿Por qué los modelos utilitaristas, no han dado respuesta a las necesidades de las poblaciones donde han sido aplicados? Lógicamente estas preguntas no se van a resolver en el presente escrito, pero si colocan sobre la mesa el debate para cuestionarse y repensar dichos modelos capitalistas, puesto que el derrumbe de las economías planificadas, no significa el éxito de las economías de libre mercado, como demostración de ello basta con mirar la crisis de Estados Unidos y de Europa a comienzos de la segunda década del siglo XXI.

En tiempos de globalización, donde hay compañías que tienen más poder supranacional que algunos países, cabe preguntar. ¿La actual crisis es responsabilidad de los Estados por no intervenir y si brindar todas las condiciones para el libre mercado? ¿Es de los empresarios por abusar de esa libertad y entrar en el codicioso juego de la especulación? ¿Más que crisis económicas, sociales y políticas: hay crisis de valores y de principios éticos? ¿Cómo hacen los países para ser autónomos en la implantación de sus modelos económicos? ¿Es problema de los modelos económicos adoptados, o problema de su interpretación y aplicación? ¿La felicidad y la prosperidad es problema individual o colectivo? ¿Es responsabilidad del individuo, del Estado o de las organizaciones? ¿Vale la pena tratar de contestar las anteriores preguntas, cuando las decisiones económicas de los Estados (especialmente los pobres) les vienen dictados por organizaciones poderosas como el Banco Mundial o el Fondo Monetario Internacional?

Pero precisamente de este poder que tienen las grandes organizaciones o las organizaciones en su conjunto, les deriva una gran responsabilidad para con sus diferentes grupos de interés donde las transacciones no sean de suma cero; por tanto, deben generar: mayor utilidad para los inversionistas, mejores condiciones para los trabajadores, cumplimiento con sus proveedores, excelentes relaciones con la comunidad 
donde están insertas, comercio justo, impuestos para el gobierno. Es decir, que la relación coste / beneficio sea favorable al conjunto de la sociedad. En una sociedad de organizaciones en alta competencia, hoy más que nunca, el principio de eficiencia necesariamente tiene que prevalecer, principio en el cual subyace la filosofía utilitarista: el mayor bien para el mayor número, o el mayor beneficio al menor coste. (Fontrodona, sin fecha). Esencialmente, esto es lo que se ha venido denominando en los últimos años como Responsabilidad Social Empresarial - RSE - que por supuesto se mide con indicadores cuantitativos: siempre presente el original principio utilitarista. Aquí cabe anotar el sentido en que se exalta la RSE:

«El impacto positivo en cada una de estas variables incide directamente en un incremento de la rentabilidad de la empresa; producto de ver la RSE con una nueva óptica, variando el enfoque tradicional de carácter filantrópico, donde las empresas responden a la comunidad en la cual trabajan apoyándola mediante acciones caritativas, hacia un nuevo enfoque donde la RSE debe ser parte integral de la estrategia de negocios $y$, por lo tanto, debe planificarse y cuantificarse con el objetivo de aumentar la rentabilidad y contribuir simultáneamente al logro de los objetivos estratégicos de las empresas» (Pesce, 2005).

Lo enunciado anteriormente, ¿es el verdadero sentido o el sentido original de al RSE? Entonces las RSE, ¿es para favorecer los intereses de la sociedad o para aumentar las utilidades de las empresas a ultranza? ¿Es para que las organizaciones compensen o le devuelvan a la sociedad todo lo que toman de ella?

Entonces, con RSE, se dan aun más las condiciones para que el Estado no intervenga, para que la «mano invisible» se encargue de regular las fuerzas del mercado: equilibrando la ofer- ta con la demanda, así mismo los precios y las cantidades. Este no intervencionismo aplica perfectamente cuando se trata de favorecer a los grandes capitales para que tengan mercados más amplios, dejando a la deriva a los pequeños empresarios o a los empresarios de países débiles, suponiendo una competencia perfecta: no intervenir, es una forma de intervención por omisión. Pero evidentemente no hay competencia perfecta puesto que las organizaciones intervienen fuertemente el mercado cuando los gremios, los clusters o los monopolios si pueden hacer sus «jugadas», generalmente especulativas, para intervenir en el mercado. Cuenta con lobistas experimentados, para que el Estado legisle (esta vez sí es licito intervenir) a favor de sus conglomerados. Es conveniente la intervención del Estado para que el aumento anual del salario mínimo de los trabajadores no rebase los límites de la competitividad, lógicamente a favor de los inversionistas. Es conveniente intervenir cuando el poderoso sector financiero está al borde de la quiebra (por especular) y se cobran impuestos a todo el pueblo para cubrirlos, pero cuando tiene unas exageradas utilidades por el abuso a que someten a sus clientes, no es conveniente que restituya todo el dinero que tomaron de la sociedad. Es conveniente intervenir para privatizar las empresas del Estado a favor de inversionistas, aunque las tarifas le sean subidas abusivamente al conjunto de la sociedad. Es conveniente promover la flexibilización laboral y extender las horas diurnas hasta las diez de la noche para que no cause recargo nocturno en el salario de los trabajadores. Es conveniente que las potencias subsidien a sus agricultores, pero no es permitido que los países subdesarrollados lo hagan, allí si no aplica la intervención del Estado.

Como se puede apreciar, no hay coherencia en la aplicación del modelo liberal pues el Estado si interviene y cuando lo hace no es neutral, lo hace a favor de los intereses de los sectores privilegiados que ostentan el poder o la riqueza, que finalmente vienen siendo los mismos. ¿Es esto ético? 


\section{Conclusión}

Más que estudiar la rigurosidad teórica de los modelos económicos, es importante entender cuál es su dinámica y la forma pragmática en que se toman las decisiones, ya lo decía Deng Xiaoping: «no importa de qué color es el gato, sino que cace ratones» (Hu, 2005). Lo que quiere decir que siempre está de primero el resultado económico sobre el principio ético o filosófico. Resultado que es medido en sus dimensiones de rentabilidad y crecimiento económico, lógicamente tasado en términos de dinero, generalmente ignorando otras dimensiones económicas como son la tasa de desempleo y el bienestar general de la población en términos de salud, educación y vivienda. Dimensiones que no necesariamente son medibles cuantitativamente.

Por tanto, en la práctica, no hay modelos económicos puros, tampoco hay modelos buenos ni malos, es la aplicación de ellos en la que se pierde sus buenos propósitos. Es sano pensar que estos modelos han sido propuestos para llevar justicia y oportunidades a la sociedad; es la interpretación de ellos y su consecuente aplicación la que hace que sean cuestionables. Los modelos económicos llevan explícita o implícitamente un contenido ético y utilitarista, en el buen sentido de la palabra, que hace que sea favorable para todas las partes, pero éste contenido ético se desdibuja en algún momento por parte de los gestores de dichos modelos.

Mill, Smith, Keynes, Taylor, Druker, Porter, han promovido principios económicos y gerenciales convenientes para todos los estamentos de la sociedad pero, en su momento, han sido tergiversados y sus propuestas se han diluido en la ambición, la codicia y el egoísmo. Tomando como ejemplo el caso de Taylor, él estaba convencido que existía identidad de intereses entre el trabajador y el patrón y que el modelo propuesto por él, hacía que tanto el trabajador como el patrón ganaran más, pero finalmente esto no fue así: aumento la productividad y las utilidades, pero no en la misma proporción el ingreso de los trabajadores, tanto que Taylor tuvo fuertes acusaciones ante la Comisión del Congreso; llegando a declarar : «... que los empresarios deben preocuparse mucho más por la calidad de lo que producen y por la felicidad de los empleados que por la rentabilidad financiera». (Barba, 2010)

Entender: los intereses individuales como opuestos al interese general, criterios técnicos contrarios a criterios éticos, trabajador como rival del inversionista, sería entender el mundo como una disyunción, como si los opuesto se excluyeran, como si las reglas del «juego» fueran de suma cero. El pensamiento complejo (Morin, 1994), propone una relación dialógica: complementariedad y conjunción entre contrarios, la inclusión, reglas de juego «gana - gana», este sería el gran impacto de un pensamiento y ejercicio económico que siempre sea leal a los principios y a la ética.

Entonces, independientemente del modelo que se aplique, si se hacen con principios éticos, seguramente van a beneficiar todos los estamentos de la sociedad, o en otras palabras: la ética y los principios son indispensables para que los modelos económicos sean viables.

\section{Referencias}

Anderson, Kerby. (2001). Utilitarismo: el mayor bien para el mayor número. Probe Ministries Suite 2000, 2001 W. Plano Parkway - Estados Unidos de Norteamérica. Recuperado de: http://www.ministeriosprobe.org/docs/utilitarismo.html Fecha de recuperación: 15/09/2011

Aranzazu Alberto San José. (1999). Crítica de Amartya Sen a la economía utilitarista. Cuadernos de Anuario Filosófico - Serie Universitaria. Pamplona Recuperado de: http://dspace.unav.es/dspace/bitstream/10171/6090/1/86. pdf Fecha de recuperación: 15/06/2013

Barba, Álvarez Antonio. (2010). Frederick Wilslow Taylor y la administración científica: Contexto, Realidad y Mitos. Revista Gestión y estrategia. No. 38 Junio / Diciembre 2010. Recuperado de: http://administracion.azc.uam.mx/ descargas/revistagye/rv38/rev38art01.pdf Fecha de recuperación: 12/05/2013 
Bentham, Jeremy, (1781). An Introduction to the Principles of Morals and Legislation, impreso en 1781. Oxford. At the Clarendon Press. London New York and Toronto. Henry Frowde. Recuperado de: http://phi673uw.files.wordpress.com/2007/01/ bentham mills.pdf Fecha de recuperación: 15/09/2011 Crespo, Ricardo. (2004). El pensamiento ético de John Maynard Keynes. Academia Nacional de Ciencias Morales y Políticas. Instituto de Ética y Política Económica. Argentina. Octubre de 2004. Recuperado de: http:// www.ancmyp.org.ar/user/files/El\%20pensamiento-crespo.pdf . Fecha de recuperación. 28/09/2011

Fontrodona, Joan. (s.f.). El utilitarismo en la ética empresarial. Cuadernos empresa y humanismo Instituto 12. Universidad de Navarra. Recuperado de: www.unav.es/ empresayhumanismo/publicaciones/.../Cuaderno012. pd... Fecha de recuperación: 25/09/2011

Giarrizzo, Victoria. (s.f.). Economía y Felicidad: ¿Existe vínculo? Facultad de Ciencias Económicas, Universidad de Buenos Aires. Recuperado de: http://www.econ.uba. ar/www/institutos/epistemologia/marco archivos/ponencias/Actas\%20XIII/Trabajos\%20Episte/Giarrizzo trabajo.pdf Fecha de recuperación 27/09/2011

$\mathrm{Hu}$, Vicky. (2006). La reforma económica china y la creación de empresas en China. Conferencia: $\mathrm{X}$ Jornada d’Economia caixaManresa I'esperit emprendedor . 2006. Recuperado de: <http://www.uoc.edu/symposia/ caixamanresa/jornadaeconomia/2005/esp/vicky_hu.pdf > Fecha de recuperación: 15/06/2013
Mill, John Stuart, (1861). El Utilitarismo, 1861 Londres. Recuperado de : http://www.ateismopositivo.com/ John\%20Stuart\%20Mill\%20-\%20El\%20Utilitarismo.pdf Fecha de recuperación: 15/09/2011

Morin, Edgar. (1994). Introducción al pensamiento complejo. Gedisa. Recuperado de: http://www.pensamientocomplejo.com.ar/docs/files/MorinEdgar_Introduccion-al-pensamiento-complejo_Parte1.pdf Fecha de recuperación: 15/06/2013

Smith, A., (1958) [1776], La riqueza de las naciones, México: Fondo de Cultura Económica.

Pesce, Dante. 2005. Responsabilidad Social Empresarial: Un Desafío para el Comercio Internacional, Bogotá: Universidad de los Andes, Recuperado de: https:// ctp.uniandes.edu.co/Empresas/Servicios/Articulos/responsabilidad social emp.php Fecha de recuperación: 26/09/2011

Sen, Amartya. (2000)., Desarrollo y Libertad, Planeta: Barcelona.

Stiglitz, Joseph E. (2012) El precio de la desigualdad. Taurus (e-book). Recuperado de: http://www.lecturasinegoismo.com/2013/01/el-precio-de-la-desigualdad-joseph-e.html\#!/2013/01/el-precio-de-la-desigualdad-josephe.html Fecha de recuperación 12/05/2013 